\title{
Concurrent Acute Monoblastic Leukemia and Multiple Myeloma in a 66-Year-Old Chemotherapy-Naive Woman
}

\author{
Srujitha Murukutla ${ }^{\mathrm{a}}$, Swaty Arora ${ }^{\mathrm{a}}$, Vijaya Raj Bhatt ${ }^{\mathrm{a}, \mathrm{c}}$, Shiksha Kedia ${ }^{\mathrm{a}}$, \\ Muhammad Popalzai ${ }^{\mathrm{b}}$, Meekoo Dhar ${ }^{\mathrm{b}}$
}

\begin{abstract}
Concurrent acute myeloid leukemia (AML) and multiple myeloma (MM) is rare, more so in chemotherapy-naive patients. Concurrent occurrence of these two malignancies portends poor prognosis. Although anthracycline-based AML regimen, allogeneic hematopoietic stem cell transplantation, tipifarnib and bortezomib have shown promising results in small number of patients, there is a lack of established therapy. We describe a case of concurrent AML and $\mathrm{MM}$ in a 66-year-old woman and review previously published literature.
\end{abstract}

Keywords: Acute myeloid leukemia; Multiple myeloma; Chemotherapy

\section{Introduction}

Concurrent acute myeloid leukemia (AML) and multiple myeloma (MM) is rare and presents unique therapeutic challenges. Here, we describe a case of concurrent AML and $\mathrm{MM}$ and review previously published literature.

\section{Case Report}

A 66-year-old woman, complaining of fatigue, loss of appetite and 25-pound weight loss for 8 weeks, was seen in the

\footnotetext{
Manuscript accepted for publication December 18, 2013

${ }^{a}$ Department of Medicine, Staten Island University Hospital, 475 Seaview Avenue, Staten Island, New York 10305, USA

${ }^{\mathrm{b}}$ Sanford R Nalitt Institute for Cancer and Blood Related Diseases, Department of Medicine, Division of Hematology and Oncology, Staten Island University Hospital, 256 Mason Avenue, Staten Island, New York 10305, USA

${ }^{\mathrm{c} C o r r e s p o n d i n g ~ a u t h o r: ~ V i j a y a ~ R a j ~ B h a t t, ~ D e p a r t m e n t ~ o f ~ M e d i c i n e, ~}$ Staten Island University Hospital, 475 Seaview Avenue, Staten Island, New York 10305, USA. Email: vrbhatta@gmail.com
}

doi: http://dx.doi.org/10.14740/wjon722w ambulatory clinic at a different institution after detection of abnormalities in blood work. Her medical history was significant for 80 pack-year smoking, chronic obstructive pulmonary disease, hypercholesterolemia, hypertension, coronary artery disease, coronary stent placement, tonsillectomy and appendectomy. The patient had a good performance status. Family history was significant for breast cancer in her mother and esophageal cancer in her father. Her medications included isosorbide mononitrate, aspirin, simvastatin, metoprolol, inhalational ipratropium, albuterol and fluticasone.

Physical examination revealed temperature of $96^{\circ} \mathrm{F}$, blood pressure of 107/73 $\mathrm{mmHg}$, pulse of 98/min and respiratory rate of $16 / \mathrm{min}$. The patient was calm and oriented. She had pallor but no icterus, petechiae, or any other evidence of bleeding. Lymph nodes were not palpable. There was no bony tenderness. Abdominal examination did not reveal hepatosplenomegaly or any other masses. Rest of the physical examination was also unremarkable.

Laboratory tests revealed white count of $64,500 / \mu \mathrm{L}$ with $17 \%$ granulocyte, $11.9 \%$ monocyte and $6.2 \%$ lymphocyte, hemoglobin of $7.5 \mathrm{~g} / \mathrm{dL}$, platelet of $100,000 / \mu \mathrm{L}$ and serum lactate dehydrogenase level of $306 \mathrm{IU} / \mathrm{L}$. Peripheral blood smear showed 3\% blasts, $73 \%$ monocytes with some immature forms, $10 \%$ neutrophils, $2 \%$ myelocytes, metamyelocytes and promyelocytes, and $4 \%$ lymphocytes. Glucose, electrolytes, coagulation profile, renal and liver function tests were within normal limits.

A bone marrow biopsy done at the other institution revealed hypercellular bone marrow with decreased bone marrow precursor cells and infiltration with plasma cells, which were kappa restricted. Flow cytometry of the bone marrow aspirate was significant for two abnormal populations: 1) myeloid cells (27\% of non-erythroid cells) with immunophenotype suggestive of monocytic differentiation; 2) plasma cells (33\% of total cells) positive for monoclonal IgG, kappa-restricted, positive for CD38, CD117 and CD56 consistent with MM. The patient was referred to our institution for further management.

Peripheral blood smear at our institution showed 12\% blasts with Auer rods. A repeat bone marrow biopsy showed hypercellular bone marrow $(70-80 \%)$ with $50-60 \%$ blasts, decreased megakaryocytes, maturing myeloid and erythroid 


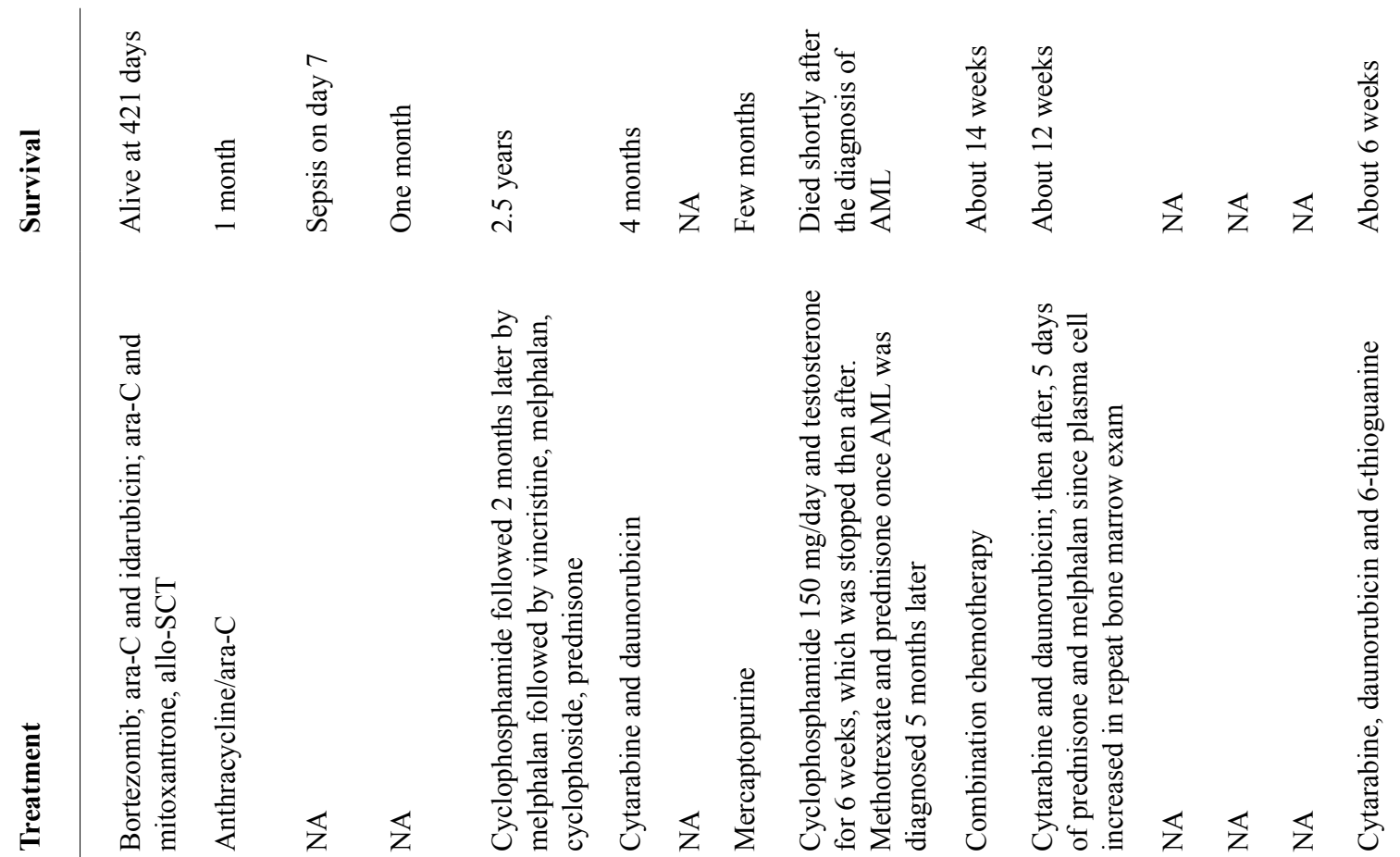

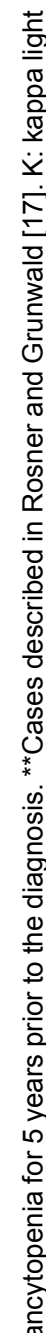

\section{క్ర}

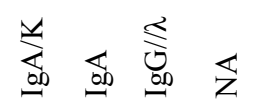

$\underset{0}{\leftrightarrows}$
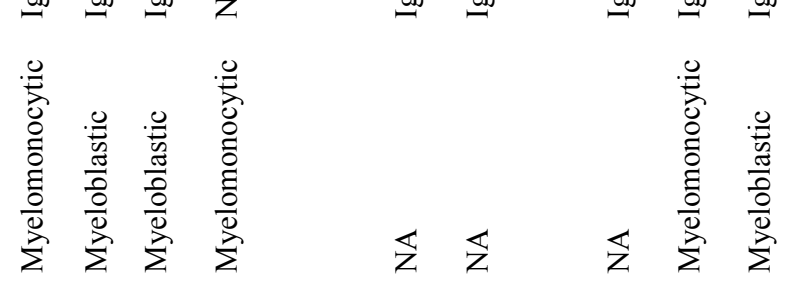

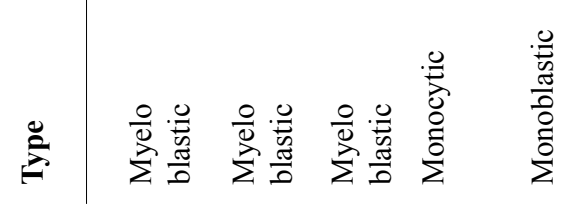

$\Sigma \Sigma \Sigma \Sigma$

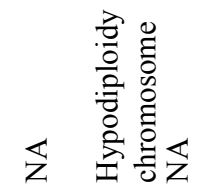

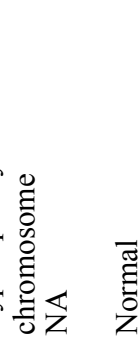

ڤั山 $\Sigma \Sigma \Sigma \Sigma \Sigma$

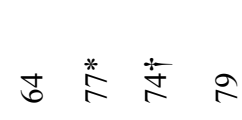

$\Sigma \Sigma$

$\Sigma \Sigma \Sigma \Sigma$

怒 $\bar{\infty}$ in

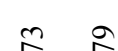

$8+\frac{1}{8}$

产

임

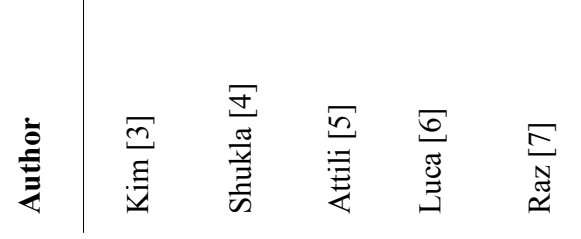

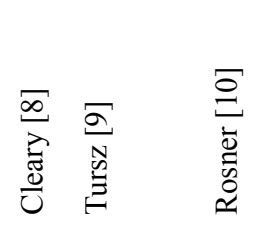

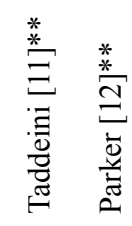

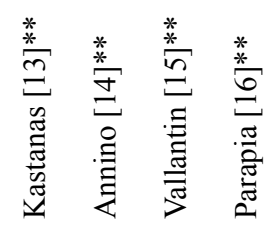


precursor cells and scattered interstitial plasma cells. Flow cytometric analysis revealed two abnormal cell populations: 1) $50-60 \%$ monoblasts with intermediate CD45 (LCA) expression with following immunophenotype: CD11c+, CD13+ (dim), CD33+, CD64+, CD14+/- (subset), CD38+, $\mathrm{CD} 4+, \mathrm{CD} 7+$ (dim), CD56+ (variable), CD117- and CD34; 2) 1-2\% atypical plasma cell population: CD38+ (bright), CD138+, CD56+. Karyotyping revealed normal female (46, $\mathrm{XX}$ ). Immunohistochemical studies revealed monoclonal kappa light chain restricted plasma cells. Fluorescent in situ hybridization was negative for any chromosomal aberrations. Serum protein electrophoresis revealed total protein of $6.5 \mathrm{~g} / \mathrm{dL}$ with immunofixation positive for monoclonal free kappa light chain band. Serum free kappa and lambda levels were $2820 \mathrm{mg} / \mathrm{L}$ and $18.2 \mathrm{mg} / \mathrm{L}$ respectively, with serum free kappa/lamda ratio of 154.9. Urine protein electrophoresis revealed monoclonal protein level of $1,901 \mathrm{mg} / 24 \mathrm{~h}$ with immunofixation positive for monoclonal free kappa light chain band. She had serum IgG of $821 \mathrm{mg} / \mathrm{dL}$, IgM of $76 \mathrm{mg} / \mathrm{dL}$ and $\operatorname{IgA}$ of $131 \mathrm{mg} / \mathrm{dL}$. Thus, a diagnosis of concurrent AML and asymptomatic MM was established. Multiple gated acquisition study revealed left ventricular ejection fraction of $60 \%$ with normal ventricular wall motion. A skeletal survey was negative.

The patient was started on allopurinol and hydroxyurea, and the latter was subsequently stopped once chemotherapy was initiated. She was induced as an in-patient with 7-day low-dose cytarabine $\left(100 \mathrm{mg} / \mathrm{m}^{2} /\right.$ day $)$ and 3-day idarubicin $\left(12 \mathrm{mg} / \mathrm{m}^{2} /\right.$ day).

Her hospitalization was complicated by Clostridium difficile diarrhea, neutropenic sepsis, and hospital acquired pneumonia secondary to Stenotrophomonas maltophila and vancomycin resistant Enteroccocus faecium. She was aggressively treated in intensive care unit but she developed multiorgan failure and died on hospital day 23 .

\section{Discussion}

AML in a patient with MM is very rare and often occurs after chemotherapy for MM [1,2]. Concurrent AML and MM in chemotherapy-naive patients is extremely rare with only few cases reported in the literature (Table 1) [3-17]. Acute myeloblastic or myelo-monocytic leukemia are the most common AML sub-types encountered in this setting. Postulated mechanisms to explain the concurrent occurrence of AML and $\mathrm{MM}$ include disorder of multi-potent stem cell $[8,18]$, exposure to common risk factors [6], leukemic or myeloma cells stimulating proliferation of bone marrow cells with subsequent development of a second hematologic malignancy [7] or AML occurring co-incidentally while monoclonal gammopathy of undetermined significance is progressing to MM [3].

Reactive plasmacytosis is common in AML patients with an incidence of up to $6 \%$ and can be associated with monoclonal paraproteins in the absence of other components of the diagnostic criteria of MM [19]. Therefore, it is important to rule out reactive plasmacytosis before making a diagnosis of concurrent AML and MM.

Given the rarity of the disease, there has been no established treatment and hence, prognosis remains extremely poor. Patients are often treated with therapy for AML since AML is more aggressive and anthracyclines are effective against $\mathrm{MM}$ as well. There are two reported cases of concurrent MM and AML in chemotherapy-naive patients, who had better outcomes compared to other patients. Raz et al describe a 68-year-old man who was diagnosed in 1978 with MM and AML without any "bizarre chromosomal changes" on cytogenetic studies. The patient was initially treated with cyclophosphamide with disappearance of monoblasts. The disease, however, recurred in 2 months when he was treated with melphalan without success and thereafter with combination chemotherapy consisting of vincristine, melphalan, cyclophosphamide and prednisone. This resulted in the disappearance of monoblasts as well as significant decline in the serum level of paraprotein. Six months later, unfortunately he developed end-organ damage related to $\mathrm{MM}$ and became unresponsive to chemotherapy. He died of septic shock and severe bleeding tendency 2.5 years from the time of initial diagnosis [7]. Kim et al describe a 51-year-old previously healthy man who was diagnosed with concurrent MM and AML with complex cytogenetics and immunoglobulin heavy chain rearrangement. The patient was initially treated with one-cycle of bortezomib and then with cytarabine (araC) and idarubicin, along with bortezomib. This was followed by re-induction with mitoxantrone and high-dose ara- $\mathrm{C}$ and mitoxantrone (HAM regimen) for induction failure. Because of incomplete response, the patient subsequently went on receiving myeloablative allogeneic hematopoietic stem cell transplantation (allo-SCT) after conditioning with busulfan and cyclophosphamide. The patient remains to be diseasefree and well at 421 days post-SCT [3]. There has been some promising result in a preclinical study in which tipifarnib and bortezomib have been shown to be synergistic in MM and AML cell lines [20]. Although the usefulness of these therapies needs further evaluation, because of the rarity of the disease, it will require an international registry to be able to do so.

\section{Conclusion}

Although very rare, AML and MM can present simultaneously even in chemotherapy-naive patients. Concurrent occurrence of these two malignancies portends poor prognosis. Anthracycline-based AML regimens are often used because AML is more aggressive and anthracyclines are effective against $\mathrm{MM}$ as well. Allogeneic stem cell transplantation as well as tipifarnib and bortezomib have shown promising re- 
sult.

\section{Financial Support}

None.

\section{Conflict of Interest}

None.

\section{References}

1. Kyle RA, Pierre RV, Bayrd ED. Multiple myeloma and acute myelomonocytic leukemia. N Engl J Med. 1970;283(21):1121-1125.

2. Bergsagel DE, Bailey AJ, Langley GR, MacDonald RN, White DF, Miller AB. The chemotherapy on plasma-cell myeloma and the incidence of acute leukemia. $\mathrm{N}$ Engl J Med. 1979;301(14):743-748.

3. Kim D, Kwok B, Steinberg A. Simultaneous acute myeloid leukemia and multiple myeloma successfully treated with allogeneic stem cell transplantation. South Med J. 2010;103(12):1246-1249.

4. Shukla J, Patne SC, Singh NK. Simultaneous appearance of dual malignancies of hematopoietic systemmultiple myeloma and acute myeloid leukemia. Indian J Pathol Microbiol. 2008;51(1):118-120.

5. Attili S, Lakshmiah KC, Madhumati M. Simultaneous occurrence of multiple myeloma and acute myeloid leukaemia. Turk J Haematol. 2006;23:209-211.

6. Luca DC, Almanaseer IY. Simultaneous presentation of multiple myeloma and acute monocytic leukemia. Arch Pathol Lab Med. 2003;127(11):1506-1508.

7. Raz I, Polliack A. Coexistence of myelomonocytic leukemia and monoclonal gammopathy or myeloma. Simultaneous presentation in three patients. Cancer. 1984;53(1):83-85.

8. Cleary B, Binder RA, Kales AN, Veltri BJ. Simultaneous presentation of acute myelomonocytic leukemia and multiple myeloma. Cancer. 1978;41(4):1381-1386.
9. Tursz T, Flandrin G, Brouet JC, Briere J, Seligmann M. Simultaneous occurrence of acute myeloblastic leukaemia and multiple myeloma without previous chemotherapy. Br Med J. 1974;2(5920):642-643.

10. Rosner F, Grunwald H. Multiple myeloma terminating in acute leukemia. Report of 12 cases and review of the literature. Am J Med. 1974;57(6):927-939.

11. Taddeini L, Schrader W. Concomitant myelomonocytic leukemia and multiple myeloma. Minn Med. 1972;55(5):446-448.

12. Parker AC. A case of acute myelomonocytic leukaemia associated with myelomatosis. Scand J Haematol. 1973;11(4):257-260.

13. Kastanas GA, Papaioannou CA, Nathena HA. Multiple myeloma and acute leukemia. Acta Haematol. 1979;62(2):78-80.

14. Annino L, Martino P, Barsotti P, Serra P, Marinozzi V, Mandelli F. Multiple myeloma and acute myelomonocytic leukemia: simultaneous occurrence without previous chemotherapy. Acta Haematol. 1980;64(4):195-200.

15. Vallantin X, Tobelem G, Daniel MT, Flandrin G, Brouet JC, Bernard J. [Myeloma together with acute myeloblastic leukaemia in an untreated patient (author's transl)]. Nouv Presse Med. 1980;9(35):2559-2560.

16. Parapia L, Abbott CR, Masters G, Roberts BE. Simultaneous presentation of acute myelomonocytic leukaemia and multiple myeloma. Acta Haematol. 1982;68(2):153156.

17. Rosner F, Grunwald HW. Simultaneous occurrence of multiple myeloma and acute myeloblastic leukemia: fact or myth? Am J Med. 1984;76(5):891-899.

18. Naparstek E, Leiserowitz R, Gamliel H, Polliack A. Simultaneous presentation of plasma cell and monocytic leukemia with a subacute clinical course. Acta Haematol. 1982;68(3):249-255.

19. Rosenthal NS, Farhi DC. Reactive plasmacytosis and lymphocytosis in acute myeloid leukemia. Hematol Pathol. 1994;8(1-2):43-51.

20. Yanamandra N, Colaco NM, Parquet NA, Buzzeo RW, Boulware D, Wright G, Perez LE, et al. Tipifarnib and bortezomib are synergistic and overcome cell adhesionmediated drug resistance in multiple myeloma and acute myeloid leukemia. Clin Cancer Res. 2006;12(2):591599. 